\title{
Infrared Radiation Calculation and Simulation of Exhaust Smoke for Armored Vehicle
}

\author{
Qingguo Luo ${ }^{\text {a }}$, Yao Zhao ${ }^{\text {b }}$, Jun Lu, Xin Liu \\ Vehicle Engineering Department, Army Academy of Armored Forces, Beijing 100072, China. \\ a946161308@qq.com,b644041689 @qq.com
}

Keywords: tank exhaust, band model, heat flow field, smoke exhaust.

\begin{abstract}
In order to study the infrared radiation characteristics of the tank exhaust, a numerical model of the tank exhaust heat flow field is established. The distribution of the flow field is analyzed and calculated, and the distribution of the temperature field of the smoke exhaust is obtained. Using spectral band model method, we calculate the radiance of exhaust gas and conduct thermal image simulation. The comparison between the calculated value of radiance and the measured value shows that the model and method can get more accurate results and can be applied to the infrared thermal image simulation of tank exhaust.
\end{abstract}

\section{Introduction}

The infrared radiation of the tank is the source of its detection by infrared detectors and destroyed by infrared guided weapons. When the tank is running at high speed, the infrared radiation of the armor plate of the power cabin becomes an important signal to be detected by the infrared detector. Besides, the thermal characteristics produced by engine exhaust pipes and exhaust pipes, artillery tubes, track and ground friction are also easy to be detected. The infrared radiation of tanks has been studied in China, but there are few studies on the calculation and Simulation of tank exhaust. The American army has done a lot of research on the flow characteristics and thermal characteristics of the exhaust gas from the military ground vehicles and began to develop a model to simulate the temperature signal of the exhaust gas from the ground vehicles, so as to predict the infrared radiation characteristics of the vehicle exhaust and display the computer image. We can see that it is very important to study the infrared radiation characteristics of tank exhaust. Therefore, the temperature and radiance of tank exhaust will be calculated and simulated in this paper.

\section{Numerical Model of Heat Flow Field}

\subsection{Mathematical Model}

The flow of tank exhaust in the exhaust pipe is very complicated. In order to study the flow state of gas outside the exhaust, this paper simplifies it into three-dimensional steady flow. The air flow field in the calculated region is described by a three-dimensional turbulent compressible air flow and heat transfer control equation, which is composed of the mass conservation equation, the momentum conservation equation, the energy conservation equation, the chemical component transmission equation and the complete gas state equation.

\subsection{Geometric Modeling}

The calculation area of heat flux field of tank exhaust is composed of two parts: the calculation area of the flow field inside the smoke exhaust pipe and the calculation area of the exhaust pipe external flow field. The structure of tank exhaust pipe in this paper is circular inlet, which is expanded to rounded rectangle outlet. The three-dimensional geometry model of exhaust pipe is constructed by software Proe, as shown in Figure 1.

The calculation area of exhaust pipe external flow field is the calculation area after the exhaust gas is discharged from the exhaust pipe. In order to set up boundary conditions conveniently, the calculation area of exhaust pipe external flow field is taken as cylinder area. Figure 2 is the whole area for the calculation of the whole heat flow field. The determination area of exhaust pipe external 
flow field is determined by multiple numerical calculation to determine the length and radius of cylinder area.

\subsection{Mesh Generation}

The mesh geometry of the area of heat flux calculation for tank exhaust is meshed by software. The degree of mesh density varies with location, which is mainly determined by the accuracy of numerical computation and the computational resources needed. For the calculation area of tank exhaust flow field, a fine mesh is divided in the near wall area of exhaust pipe. In other areas, mesh adaptation technology is used to adjust mesh density. In order to ensure the grid independence of the area grid model of the tank exhaust heat flow field, the grid size of the calculation area of the flow field in the exhaust pipe is changed under the condition of determining the calculation area of the outflow field of the exhaust pipe, so that the results of the calculation of temperature, velocity and pressure are hardly affected when the grid size is further changed.

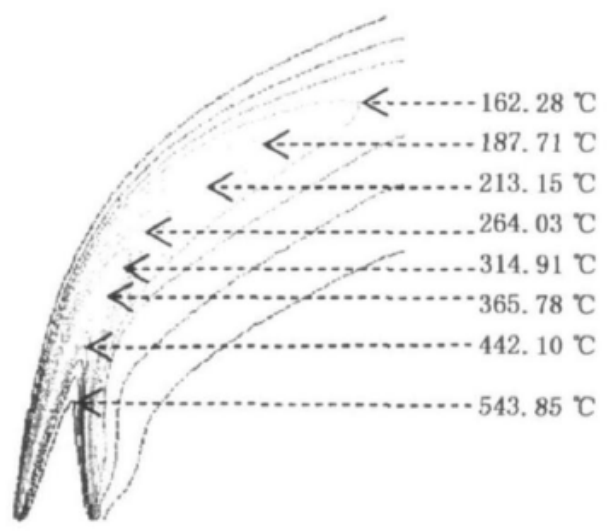

Figure 1. Temperature field of exhaust

\subsection{Setting Boundary Conditions}

The boundary conditions for the numerical calculation of the heat flow field of exhaust smoke include the inlet boundary condition of mass flow, the boundary condition of the pressure inlet, the boundary condition of the pressure outlet and the boundary condition of the wall. The boundary conditions are set as shown in Figure 3.

(1) Flow inlet boundary conditions: gas mass flow, temperature, turbulence intensity, dissipation rate and mass fraction of smoke components are set, and the gas mass flow and temperature are calculated by the calculation of the working process software of the internal combustion engine.

(2) pressure inlet boundary condition: pressure is calculated according to vehicle speed and wind speed, the temperature is air temperature, and the mass fraction of components is air mass fraction.

(3) the pressure outlet boundary condition: the pressure after full expansion should be equal to the atmospheric pressure, and the temperature is the atmospheric temperature.

(4)the wall boundary condition: the standard wall function is applied, and the thermodynamic boundary is the first boundary condition, namely the fixed wall temperature.

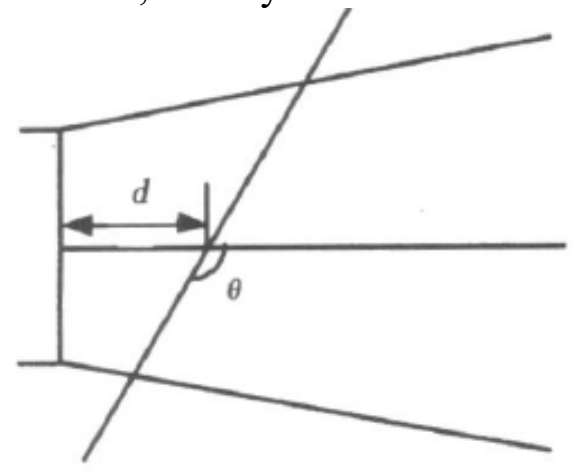

Figure 2. Schematic diagram of smoke exhaust 


\subsection{The Discrete and Solution of the Equation}

The governing equations are discretized by finite volume method and staggered grid system. Diffusion terms and source terms are based on the two-order upwind scheme. The velocity and pressure coupling use pressure correction equation, continuous equation, momentum equation, energy equation chemical component transfer equation to carry out sub relaxation, relaxation factor is adjusted gradually in the calculation process. In order to ensure the stability and convergence of the numerical calculation, the convergence standard of the numerical calculation of the heat flow field of the tank exhaust is set. When the iterative calculation reaches the set of convergent standards, the iterative convergence and the end of the calculation are considered.

\subsection{An Example of the Temperature Calculation of the Heat Flow Field}

In view of the above conditions, first, according to the type of diesel engine, make corresponding assumptions, and establish its working process calculation model. The exhaust gas temperature and mass flow at the outlet of the exhaust pipe can be obtained by calculation. Then as a known condition, the heat flow field of tank exhaust is calculated according to the above numerical calculation model. The results of the temperature of the heat flow field are shown in Figure 2 and figure 3.

From Figure 2 and figure 3, it can be seen that the flue gas temperature of the exit section of the exhaust pipe can be up to $543{ }^{\circ} \mathrm{C}$, and there is a region with a constant and maximum temperature at the exit of the exhaust pipe. The infrared radiation characteristics of the whole exhaust smoke are largely determined by the radiation in this area.

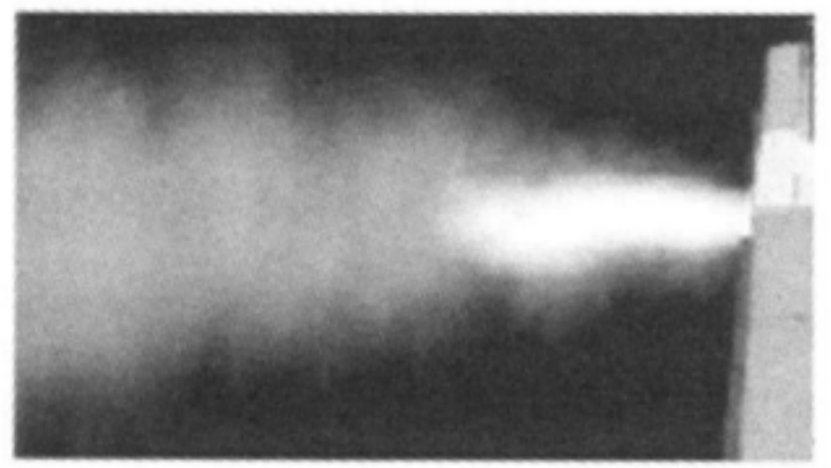

Figure 3. Schematic diagram of infrared radiation for smoke

\section{Infrared Radiation Calculation of Tank Exhaust}

Assuming that the combustion of diesel fuel is complete combustion, the typical smoke selective exhaust is mainly in the emission band. In order to calculate the radiation of smoke exhausting along any direction, the way of determining the direction from the distance between the axis and the exhaust port is adopted, as shown in Figure 6.

After the temperature distribution of smoke exhaust and the pressure distribution of each component are known, the radiance of smoke exhaust along any line of sight can be approximately calculated by using the spectral band model. The non-uniform smoke emission is considered as the radiation superposition of locally homogeneous gas. The key point of the calculation is to divide the smoke exhaust part into a sub layer with uniform temperature and pressure. In order to calculate the radiation of each layer of each layer, the spectral transmittance of each layer is calculated. The radiance of the total spectral radiance along the line of sight is calculated using the transmittance of each layer, and the radiance of the radiation band is obtained at the end of the number and the interval of the wave number. The main calculation steps are as follows:

1.the smoke extraction part of any line of sight is divided into a uniform layer of temperature and component pressure, and the same layer is approximately isothermal and isobaric. The temperature of each layer is pressure.

2.determine the waveband range of the main emission bands of infrared emission components and carbon dioxide. 
3.the thickness calculation under the standard state. Calculate the thickness of each component in each layer.

4. the absorption coefficient and line density of Xian spectrum are calculated by interpolation. These two quantities are all within the range of wave number interval 0- 25 .

5. the calculation of the transmittance. Considering the collision broadening and Doppler broadening of spectral lines, the spectral transmittance of each layer is calculated from the influence of spectral width and optical thickness.

6.use the radiative transfer equation to calculate the spectral radiance of each layer. According to the radiative transfer equation, the spectral radiance of a wave number in each layer is calculated according to the transmittance of each layer.

7.calculate the radiance of a line of sight and get the sum of layers and wave numbers by spectral radiance.

\section{Test of Infrared Radiation Characteristics of Exhaust Smoke}

In the experimental test, the wind speed is $2.54 \mathrm{~m} / \mathrm{s}$, the air temperature is $-5.18^{\circ} \mathrm{C}$, the atmospheric pressure is $101.5 \mathrm{kPa}$, the atmospheric transparency rate is 0.75 , the sun height angle is 70 degrees, the test condition is the engine running tank still, its rotational speed is $2000 \mathrm{r} / \mathrm{min}$, the throttle opening is 800 degrees, the inlet and exhaust blinds are all open. Because the highest temperature of smoke exhaust $\mathrm{H}_{2} \mathrm{O}$ is below $600{ }^{\circ} \mathrm{C}$, the minimum infrared radiation can be ignored. The field of view of the test includes the cavity of the exhaust pipe, so the radiance of the smoke tube cavity is considered when calculating the infrared radiance of the smoke exhaust. The comparison between the test value and the calculated value is shown in Table 1.

From table 1, we can see that the relative error is $10.84 \%$, which can meet the needs of engineering design. In addition, in a certain range, with the increase of ambient temperature, the radiance of exhaust smoke increases gradually, because the temperature field of the tank exhaust is closely related to the working conditions of the tank engine, and the temperature of the environment affects the temperature of the engine intake, so it will have an effect on the temperature of the exhaust. However, the influence of solar radiation on smoke exhaust is very small, because solar radiation has little influence on the heat flux of smoke components. Besides, with the increase of ambient wind speed, the radiance of exhaust gas will also gradually decrease. Because under the action of wind speed, the convective heat transfer between exhaust gas and air is aggravated, and the temperature of exhaust gas has been reduced, resulting in the decrease of radiation intensity of exhaust gas.

\section{Infrared Thermal Image Simulation of Smoke Exhaust}

According to the previous calculation results, the relationship between radiation brightness and gray level is established to make the gray value of the image represent the radiation brightness. The infrared thermal image of the tank smoke is simulated, and the simulation results are shown in figure 7.

It can be seen that because the temperature of smoke exhaust is very high, the radiation brightness is high. While the rest of the tanks are not calculated in this article the radiation brightness, but according to calculation results in the group in the past, the tank to produce all the heat out of the smoke pipe outlet of the exhaust smoke temperature is the highest, and close to the exhaust port of armor plate temperature significantly higher than the other plates. These apparent thermal characteristics of tanks and background infrared radiation characteristics of the contrast between the larger, sharp contrast to the tank heat map image structure, make the tank was found to be extremely easily, recognition and tracking. 


\section{Conclusion}

In this paper, the heat flow field of tank smoke is analyzed deeply. A complete numerical calculation model of exhaust heat flow field is presented. Based on the calculated values of carbon dioxide radiance and the measured data are compared and verified. The accuracy of the method and the model, the realistic image and the actual imaging device There is good consistency. In addition, the infrared radiation of tank smoke is calculated. For the sake of simplicity, let's assume that the smoke emission group is divided into carbon dioxide and the water did not consider the carbon smoke particles, but the actual smoke composition comparison. Complexity, to obtain more realistic simulation results, remains to be further deeply research.

\section{References}

[1]. Suarex M J, Chou M D. Technical report series on global modeling and data assimilation. Volume 3: An efficient thermal infrared radiation parameterization for use in general circulation models[C]// Unknown. Unknown, 1994:1603-1609.

[2]. Forget F, Pierrehumbert R T. Warming early Mars with carbon dioxide clouds that scatter infrared radiation [J]. Science, 1997, 278(5341):1273-6.

[3]. Miller W H. A classical/semiclassical theory for the interaction of infrared radiation with molecular systems [J]. Journal of Chemical Physics, 1980, 72(1):788-789.

[4]. Suarex M J, Chou M D. Technical report series on global modeling and data assimilation. Volume 3: An efficient thermal infrared radiation parameterization for use in general circulation models[C]// Unknown. Unknown, 1994:1603-1609.

[5]. Zheng L. Infrared radiation absorbing articles and method of manufacture [J]. 2018.

[6]. Rohacs J, Jankovics I, Gal I, et al. Small Aircraft Infrared Radiation Measurements Supporting the Engine Airframe Aero-thermal Integration [J]. Periodica Polytechnica Transportation Engineering, 2018.

[7]. Tsuda S, Yamaguchi S, Kanamori Y, et al. Spectral and angular shaping of infrared radiation in a polymer resonator with molecular vibrational modes[J]. Optics Express, 2018, 26(6):6899.

[8]. Eskandari J, Kermani A M, Kouravand S, et al. Design, fabrication, and evaluation a laboratory dry-peeling system for hazelnut using infrared radiation[J]. LWT- Food Science and Technology, 2018, 90 . 